\title{
High resolution computed tomography and classification of children with interstitial lung diseases
}

\author{
M.L. He, H. Lai, Y. Cao, Y.Z. Shuai and S.C. Yu \\ Department of Radiology, Chengdu Women and Children Center Hospital, \\ Chengdu, China \\ Corresponding author: M.L. He \\ E-mail:mlsccn@126.com
}

Genet. Mol. Res. 13 (2): 4241-4251 (2014)

Received May 3, 2013

Accepted January 13, 2014

Published June 9, 2014

DOI http://dx.doi.org/10.4238/2014.June.9.9

\begin{abstract}
High resolution computed tomography (HRCT) was used to classify children with interstitial lung diseases (ILD). Sixty children with ILD underwent HRCT in supine position under freerespiratory conditions during scanning. Children under 5 years old were sedated with chloral hydrate and the scanning scope was from the lung apex to the diaphragm. In children older than 5 years old, scans were obtained at three levels: aortic arch, tracheal carina, and $1 \mathrm{~cm}$ above the right diaphragm. Five infectious patients were followed up. Two experienced radiologists read the films blindly to observe the type and distribution of ground-glass opacities and bronchovascular bundle abnormalities. Bronchovascular bundles were thick in 49 patients, and were thick and stiff in 27 patients. Of the 41 infectious patients, 39 showed thickened bronchovascular bundles, and 26 showed thick and stiff bronchovascular bundles. Of the 19 non-infectious patients, bronchovascular bundles were thickened in 10 patients, and were thick and stiff in 1 patient. Fortyone patients showed lobular ground-glass opacity (32 infectious, 9 non-infectious). Twenty-seven patients showed both bronchovascular
\end{abstract}


bundle abnormality and lobular ground-glass opacity (20 infectious, 7 non-infectious). Eighteen patients showed patchy or mosaic ground-glass opacity (16 infectious, 2 non-infectious). There were 4 cases of bronchiectasis. HRCT is the first non-invasive diagnostic method for children with ILD, and its different manifestations can be classified. In early manifestation, bronchovascular bundles were abnormal and complicated with lobular ground-glass opacity. Patchy ground-glass opacity was the most common manifestation, and appeared to be difficult to disappear. Bronchiectasis indicated that the disease is irretrievable.

Key words: Children; Interstitial lung disease; High resolution CT; Disease classification

\section{INTRODUCTION}

Children's interstitial lung diseases (ILD) are a group of genetically heterogeneous diseases featuring diffuse lung infiltration and restrictive lung diseases (Hartl and Griese, 2005), including interstitial pneumonia and other changes of the lung caused by systemic diseases. They are common clinical diseases that seriously affect the health and development of children, and their incidence rates increase every year. ILD can generally be defined by the following two symptoms: both respiratory system symptoms and diffuse infiltration changes are apparent in chest X-rays, and pulmonary function tests show restrictive ventilation disorder and gas exchange impairment. Patients generally have one of the above two symptoms for more than 3 months (Gross and Hunninghake, 2001). The clinical manifestations of these kinds of diseases include hypoxemia, cyanosis, acropachia, and developmental retardation, among others (Hilman, 1998; Fan and Langston, 2002). There is no authoritative report about the incidence of children's ILD, although some studies have indicated that the incidence is 3.6 per million (Dinwiddie et al., 2002). A German study reported that the annual incidence of ILD was 1.32 per million (Griese et al., 2009). With the advent of updated testing technology and continuous improvements at the diagnosis level, reports of the incidence of ILD in Chinese domestic children have also increased in recent years.

Computed tomography (CT) has an important diagnostic significance for ILD (Grenier et al., 1991; Seely et al., 1997). At present, imaging technology, including high resolution CT (HRCT), only provides a general basis for the diagnosis of interstitial pneumonia. It can neither distinguish the severity of lesions, nor reflect the relationship between clinical symptoms and pathology, and is not at all informative with respect to illustrating the effects of clinical therapy. The clinical application of HRCT improves lung imaging diagnosis at the lobular level, and is considered to be the best noninvasive test method of lung diseases (Mayo et al., 1987). HRCT can provide pathological information of pediatric lung diseases to a certain extent (Fan and Langston, 2002). Unfortunately, the ILD classification of domestic and foreign children remains controversial, and there is currently no satisfactory clinical-imagingpathological diagnosis (CRP diagnosis) type, which seriously affects the clinical diagnosis and assessment of treatment. Because clinical manifestations and pathological changes of adult ILD and child ILD are not exactly the same, many of the studies of adult ILD are not com- 
pletely suitable for cases in children with complex etiologies and clinical variations (GarciaPeña and Lucaya, 2004).

We here examined HRCT manifestations of 60 cases of children's ILD and reviewed related literature in order to enable diagnoses and types of children's ILD to be associated with clinical and pathological metrics using imaging tests. This type of classification will facilitate services that can be provided for clinical diagnosis and assessments of therapeutic effects.

\section{MATERIAL AND METHODS}

Sixty children with ILD, diagnosed by clinical, lung function, and imaging tests between September 2003 and October 2009, were recruited, including 49 males and 11 females. The youngest patient was two months old, the oldest was 13 years old, and the average age was 3.4 years. There were 41 infectious patients (15 with Mycoplasma pneumoniae pneumonia; 4 with Chlamydia trachomatis pneumonia; 3 with respiratory syncytial virus [RSV] pneumonia; 2 with concomitant Mycoplasma pneumoniae and RSV infection; 2 with pertussis; 1 each with Enterobacter cloacae pneumonia, Klebsiella pneumoniae, influenza virus, parainfluenza virus, cytomegalovirus, and infectious mononucleosis; and 9 with composite pathogen infection) and 19 noninfectious patients (10 with asthma, 3 with idiopathic pulmonary hemosiderosis, 1 with idiopathic alveolar fibrosis, and 5 with bronchopulmonary dysplasia [BPD]).

The Siemens Emoution Due 2-slice scanner was used for HRCT scanning with the following parameters: $110 \mathrm{Kv}, 50 \mathrm{~mA}, 500 \mathrm{HU}$ window width, $-700 \mathrm{HU}$ window level, and $1 \mathrm{~mm}$ layer thickness. Sick children that were less than 5 years old were sedated with $0.3-0.5$ $\mathrm{mL} / \mathrm{kg}$ chloral hydrate before scanning. Sick children took the supine position. Sick children more than 5 years of age were scanned without breathing restrictions at 4 levels of 3 regions: aortic arch, eminence and base of lung, and $1 \mathrm{~cm}$ above the right diaphragm. Children under 5 years old were scanned continuously from the apex of the lung to the costophrenic angle area. The head, neck, abdomen, and pelvic area were covered for protection.

Two highly qualified imaging diagnosticians read the films in a double-blind manner to observe differences between infectious and noninfectious ILD images. Infectious ILD showed bronchovascular bundle abnormalities, ground-glass opacities, interlobular septal thickening, bronchial wall thickening, and bronchiectasis. Five cases of infectious ILD had follow-up visits. The follow-up period ranged from 4 months to 36 months, and the average was 14 months.

\section{RESULTS}

The 60 cases analyzed in this study included 49 cases of bronchovascular bundle thickening and 27 cases of spasticity. The 41 cases of infectious ILD included 39 cases of bronchovascular bundle thickening and 26 cases of bronchovascular bundle spasticity. The 19 cases of noninfectious ILD included 10 cases of bronchovascular bundle thickening and 1 case of bronchovascular bundle spasticity. The manifestation of bronchovascular bundle spasticity was when the bronchovascular bundle radially extended from the inner side of lungs to the periphery and lost its normal tree-like morphology, which was termed a grass-like change. In some cases, even double-track signs could be seen, and the tips were prick-like (Figure 1A and B; Table 1). 

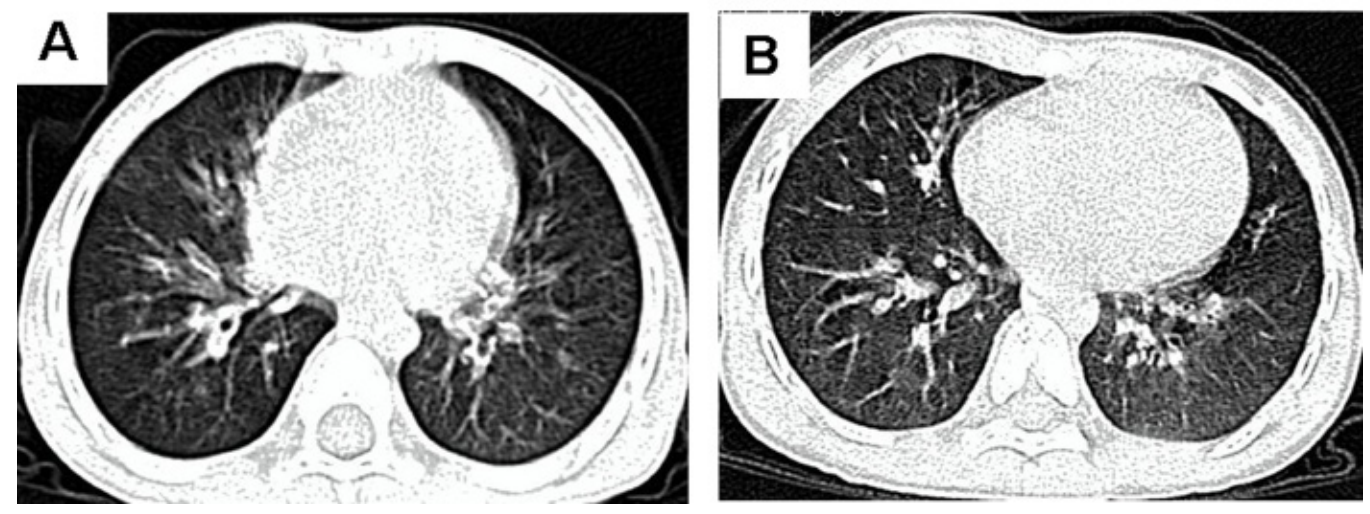

Figure 1. Male, 3 years-old, parainfluenza virus infection pneumonia. A. Three years ago. B. three years later, lobular ground-glass opacities have almost been absorbed, the signs of bronchovascular bundles vary mildly, and abnormal stiffness have also been improved.

Table 1. Contrasts of "spasticity" and "thickening" between infectious group and noninfectious group.

\begin{tabular}{lcccc}
\hline Variables observed & Infectious group (\%) & Noninfectious group (\%) & $\chi^{2}$ & $\mathrm{P}$ \\
\hline Thickening & $39(95.12 \%)$ & $10(52.63 \%)$ & 15.656 & 0.000 \\
No thickening & $2(4.88 \%)$ & $9(47.37 \%)$ & & \\
Spasticity & $26(63.41 \%)$ & $1(5.26 \%)$ & 17.7739 & 0.000 \\
No spasticity & $15(36.59 \%)$ & $18(94.74 \%)$ & & \\
\hline
\end{tabular}

There were 41 cases of lobular ground-glass opacities, including 32 cases of infectious lobular ground-glass opacities (Figure 2) and 9 cases of non-infectious lobular groundglass opacities. For infectious lobular ground-glass opacities, the severity was most obvious around the bronchovascular bundles.

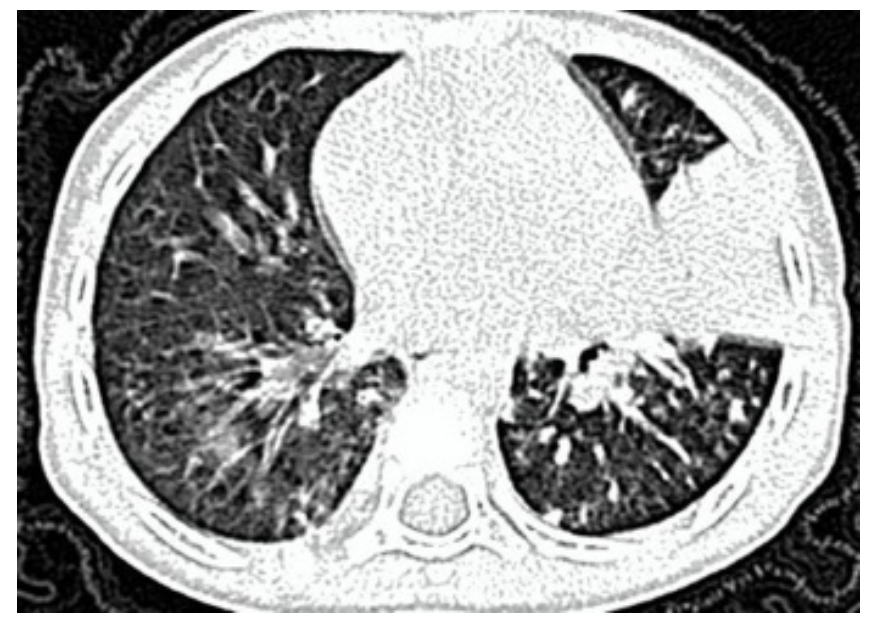

Figure 2. Male, 10 years-old, Chlamydia trachomatis infection pneumonia. 
Twenty-seven patients had both bronchovascular bundle abnormalities and lobular ground-glass opacities; 20 patients were infectious and 7 patients were noninfectious.

There were 46 cases of bronchial wall thickening; 37 cases were infectious and 9 cases were noninfectious.

There were 18 cases of large patchy ground-glass opacities; 16 cases were infectious and 2 cases were noninfectious. Thirteen of the 16 patients with infectious large patchy ground-glass opacities were complicated by lobular ground-glass opacities (Figures 3 and 4).

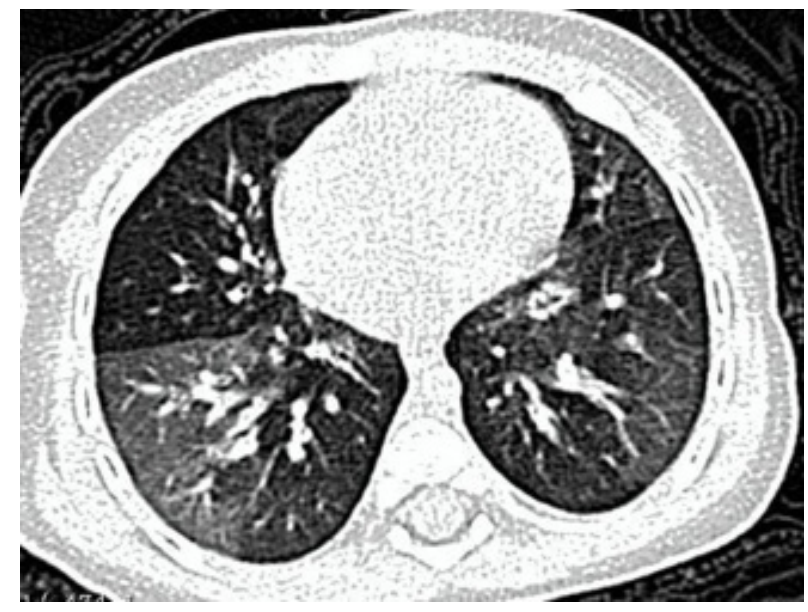

Figure 3. Male, 1 year-old, Chlamydia trachomatis infection pneumonia.

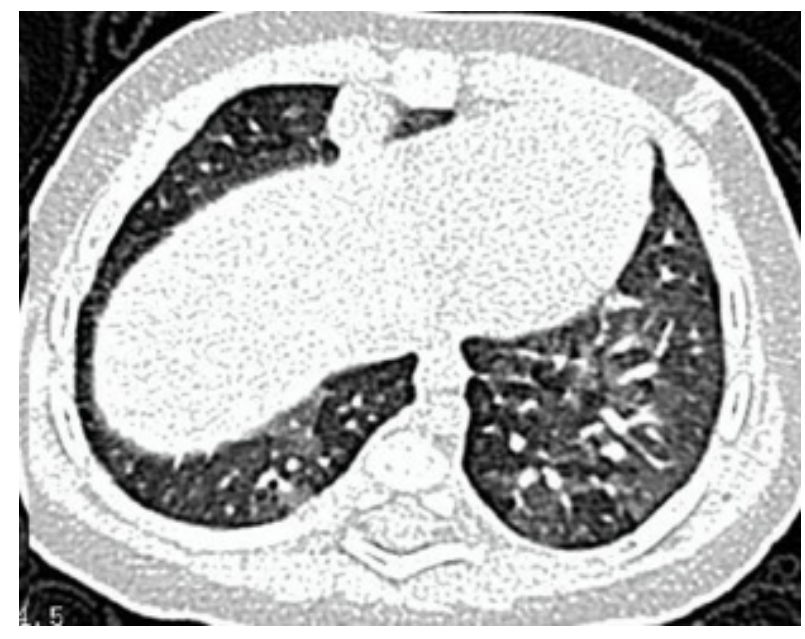

Figure 4. Female, 5 months, Mycoplasma pneumoniae infection pneumonia.

In some cases, transverse diameters of ambient bronchia were observed. If they were larger than the transverse diameters of concomitant vessels at the same level, we designated it as bronchiectasia. There were 4 cases of bronchiectasia (Figure 5); 3 cases were M. pneu- 
moniae infection and 1 case was asthma. All 4 cases were complicated by lobular ground-glass opacities, and one was complicated by patchy ground-glass opacities.

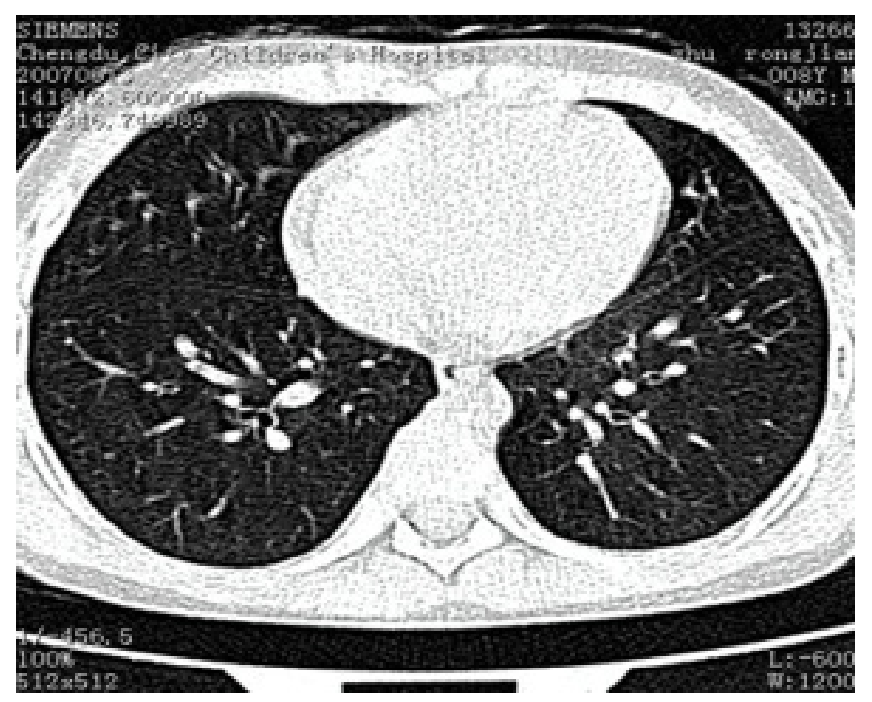

Figure 5. Male, 5 years-old, Mycoplasma pneumoniae infection pneumonia, right lower lung local bronchial columnar expansion.

There were 21 cases of lobular atelectasis or phased lung consolidation. Thirteen cases were infectious and 8 cases were noninfectious. All of them showed irregular, patchy, opaque, high-density shadows with a clear boundary in the lung field (Table 2).

\begin{tabular}{|c|c|c|}
\hline & Infectious & Noninfectious \\
\hline No axial interstitial change & 0 & 9 \\
\hline Axial interstitial type & 5 & 1 \\
\hline Axial interstitial + lobular ground-glass opacities type & $20(48.78 \%)$ & $7(36.84 \%)$ \\
\hline Patchy ground-glass opacities type & $16(39.02 \%)$ & $2(1.11 \%)$ \\
\hline Bronchiectasia & 3 & 1 \\
\hline Lobular or phased lung consolidation & $13(31.71 \%)$ & $8(42.11 \%)$ \\
\hline
\end{tabular}

HRCT follow-up was conducted on 5 patients with infectious ILD. All the 5 patients originally had bronchovascular bundle thickening and spasticity. The conditions of 4 patients did not change based on results of follow-up visits, whereas the extent of spasticity of 1 patient improved 3 years later (Figure 1B). Three of the five patients originally had large, patchy ground-glass opacities, and the condition of only one of these patients did not change based on results of follow-up visits. One patient developed large, patchy ground-glass opacities on the basis of lobular ground-glass opacities. Four of the original 5 patients had phased lung consolidation. The lung consolidation of 3 patients disappeared. The lung consolidation of 1 patient was somewhat absorbed, but a strip fibrosis shadow appeared. 


\section{DISCUSSION}

Sixty cases of children's ILD could be classified into two main types based on etiology: the infectious type and the noninfectious type. The data shown in Tables 1 and 2 indicated that the HRCT manifestations of infectious ILD and noninfectious ILD were obviously different. Although HRCT could not provide etiological diagnoses for infectious ILD, it could more intuitively reflect the imaging conditions of disease development. The HRCT manifestations of children's infectious ILD were considered to be mainly bronchovascular bundle abnormalities, lobular ground-glass opacities, large, patchy ground-glass opacities, and bronchiectasia. Each of these imaging manifestations and their correlations will be discussed in turn in the following sections.

\section{Bronchovascular bundle abnormalities}

The thickening of bronchovascular bundles is the main HRCT manifestation of infectious ILD. Clement et al. (2010) studied thin-section CT manifestations of 24 patients with $C$. pneumoniae pneumonia, and found 17 cases (70.1\%) of bronchovascular bundle thickening. In this study, the constituent ratios of "thickening" and "spasticity" of the infectious group and the noninfectious group shown in Table 1 were statistically compared, and both were statistically significant $\left(\chi^{2}=15.656, \mathrm{P}=0.000\right.$ and $\chi^{2}=17.739, \mathrm{P}=0.000$, respectively). Remarkably, 38 of the 41 patients with infectious diseases suffered from non-bacterial infections. However, 1 sick child with asthma in the noninfectious group also manifested bronchovascular bundle thickening and spasticity. Perhaps, due to repeated airway spasm and contraction, the bronchovascular bundles thickened, and even exuded inflammation. However, a history of airway infection was not excluded for this case. Children's infectious ILD are mostly attributed to primary airway infections. The infections of viruses, mycoplasma, and chlamydia first spread along the trachea and then pass through small and medium-sized bronchi, forming axial interstitial inflammation (Nambu et al., 2006). Airway mucosa and submucosa involvement caused by repeated infection is the main reason for the abnormal changes of bronchovascular bundles. Thirty-nine of the 41 patients with infectious diseases in this group had bronchovascular bundle thickening, and 26 patients had both bronchovascular bundle thickening and spasticity. Ten of the 19 patients with noninfectious diseases had bronchovascular bundle abnormalities, but only 1 patient manifested spasticity. Bronchovascular bundle changes in children with infectious ILD were mostly complicated by lobular ground-glass opacities (Table 2), which manifested as scattered punctate shadows that were mostly distributed around the abnormal axis interstitial space. Twenty of the 41 patients with infectious diseases in this group had bronchovascular bundle abnormalities, and there were lobular ground-glass opacities around them. Notably, none of the original bronchovascular bundle symptoms, including thickening and spasticity, of the 5 patients with infectious ILD who were followed-up disappeared. Moreover, only 1 patient showed improvement 3 years later (Figure 1B). This suggests that bronchovascular bundle spasticity with lobular ground-glass opacities is an early specific symptom of children's infectious ILD, reflects early pathological features of lesions, and can be regarded as a type of imaging manifestation.

\section{Lobular ground-glass opacities}

Ground-glass opacities appeared as lung parenchyma abnormal shadows in children's 
ILD following ambient interstitial changes, and were also the most common HRCT manifestation in this group (32 cases). Most of them were lobular, and manifested as scattered punctate or gathered punctate translucent shadows around the interstitial axis. The formation of groundglass opacities reflected a relatively complex pathological process and was related to the extent of pathological changes of small airway involvement. The formation mechanism of different ILD group-glass opacities is not exactly the same and can be caused by the incomplete inflation of pulmonary alveoli caused by inflammation, edema, fibrosis, or tumors (Clement et al., 2010). Ground-glass opacities of acute interstitial pneumonia (AIP) reflect the formation of transparent lung membrane (Park et al., 2007). Siegel et al. (2001) reported that lesions were distributed around bronchi and presented lobular (punctate) or patchy density-increased shadows, which was similar to tuberculous pneumonia. The lesions also had a gradient distribution characteristic in which they gradually increased from the apex to base of the lung. The lesions distribution in the present study was in accordance with previous reports, and showed an obvious gradient distribution. The formation of this characteristic distribution was related to the child's age as well as the structural anatomy of the lung and many other factors. There are significantly more mature airway branches in the lower lung than that in the upper lung, up to 22-25 levels (Long et al., 2005). The airway inflammatory reaction results in airway irritation contraction, and increased secretions in the airway amplify the airway resistance. Consequently, the pulmonary alveoli can barely inflate or inflates poorly. However, in this study, lobular ground-glass opacity rather than typical nodule shadows were observed, suggesting that lobular ground-glass opacity is the main type of children's ILD. The imaging difference of the lobular ground-glass opacity observed in children's ILD and the nodule shadows observed in adult ILD should be further investigated.

\section{Patchy ground-glass opacities}

Mosaic shadows are typical HRCT manifestations of bronchiolitis obliterans (BO) (Johkoh et al., 1999). Connective tissue disease, drug toxicity, inhalation injury, or viral pneumonia could result in this manifestation. A crazy-paving pattern is apparent in groundglass opacities, which shows a map-like distribution on HRCT and is overlapped by reticular smooth filament shadows. This pattern is generally regarded as the result of alveolar consolidation and interstitial fibrosis. In addition to pulmonary alveolar proteinosis, mosaic shadows are common in adult respiratory distress syndrome, bacterial pneumonia, AIP, and diffuse alveolar damage of usual interstitial pneumonia (UIP). Compared to desquamative interstitial pneumonia (DIP), UIP has older onset ages and obvious interlobular septal thickening shadows (Wittram et al., 2003; Bramson et al., 2005; Levine et al., 2007). However, children's ILD, especially children's infectious interstitial pneumonia, mostly led to the involvement of alveolar spaces, alveolar ducts, and small airways, whereas interlobular septal thickening was not common. The "crazy-paving pattern" was not found in patients of the present study, suggesting that the large patchy ground-glass opacities of children's interstitial pneumonia rarely manifest the crazy-paving pattern. Garcia-Peña and Lucaya (2004) carefully observed a group of children with $\mathrm{BO}$, which were classified as pneumonia, respiratory bronchiolitis, interstitial lung disease, and infiltration of histiocytes in alveolar spaces. Diagnoses were also based on the formation of visible connective tissue granulation in alveolar spaces, alveolar ducts, and bronchioles, and even changes of lumen obstruction characteristics. Of the 60 cases analyzed 
in this study, 18 manifested large, patchy ground-glass opacities, including 16 infectious cases and 2 noninfectious cases. Nine of the 16 infectious cases had a chronic cough for more than 3 months, and 7 cases had it for more than 6 months. Seventeen of the 18 cases manifested decreased lung function, a slight increase in airway resistance, and a decrease in lung compliance. The pulmonary function test resulted of 1 case was normal. Three of the 5 infectious patients who had follow-up visits manifested large, patchy ground-glass opacities. Only 1 of these cases showed no change 4 months later based on follow-up visits. Two cases showed absorption of different degrees 6 months later according to review. The condition of 1 case developed 8 months later. Patchy ground-glass opacities were manifested on the basis of lobular ground-glass opacities, and 13 of the 16 infectious patients were complicated by lobular ground-glass opacities. Therefore, we believe that the manifestation of large, patchy groundglass opacities was the result of the continued development of bronchiolitis following lobular ground-glass opacities, and reflected the clinical and pathological progress of children's ILD. In addition, it could be regarded as an imaging type of the progressive stage of children's ILD. However, changes in patchy ground-glass opacities were not irreversible; some of these patients recovered to different degrees and generally took a longer time. Typical mosaic perfusion signs were not found in any patients of this group, which might simply be a limitation of the samples of this particular study. However, mosaic perfusion signs are not as common in children's ILD as in adult ILD. Therefore, the mosaic perfusion signs of children remains to be elucidated with further HRCT investigations.

\section{Bronchiectasis}

Bronchiectasis is the unrecoverable abnormal expansion of local bronchia caused by the destruction of the elastic and muscle tissues of bronchial walls. It represents the fibrosis manifestation around diseased bronchia as well as the common final result of many pathological processes (Wittram et al., 2003; Levine et al., 2007). The intuitive reasons for bronchiectasis are a decrease in lung volume and fibrosis around lesions. Bramson et al. (2005) indicated that bronchiectasis caused by pyogenic bacteria and viral infections often occurred in the two lower lungs. Due to the occlusion of its distal bronchi, bronchiectasis usually occurs in medium-sized bronchia. There were 4 sick children with bronchiectasis in this study. The course of the disease in 3 patients was more than 6 years. The other patient was 5 years old, and the course of disease was more than 3 years (Figure 5). Four cases of bronchiectasis were complicated by lobular ground-glass opacities. One case was also combined with patchy ground-glass opacities. Results of previous studies (Brody, 2008; Farrell et al., 2009) and the present study suggest that bronchiectasis is the first indicator of the final pathological stage of children's ILD and is an unrecoverable image manifestation.

\section{Lobular pulmonary consolidation}

Lobular or phased pulmonary consolidation is the image manifestation of alveolar inflammation. Children's ILD often involves the alveoli, which causes lung parenchyma inflammation. Usual alveolar inflammatory consolidation can be absorbed. Intercurrent alveolar inflammatory consolidation of children's infectious ILD can also be absorbed, although the absorption process is not as long as that of interstitial inflammation. Twenty-one of the 60 
patients in this group had lobular or phased consolidation, including 13 infectious cases and 8 noninfectious cases. Pulmonary consolidation shadows of 5 infectious cases disappeared in follow-up visits. With the control of patients' conditions and absorption of bleeding in pulmonary alveoli, pulmonary consolidation of noninfectious cases, such as pulmonary hemosiderosis, disappeared spontaneously. These results suggest that pulmonary consolidation should not be classified as an imaging type of children's ILD.

Different causes of noninfectious ILD lead to different pathological changes, developments, and prognoses. Therefore, it is difficult to uniformly classify and phase HRCT manifestations. There were 19 cases of noninfectious ILD, including 10 cases of asthma, 5 cases of BPD, 3 cases of pulmonary hemosiderosis, and 1 case of idiopathic alveolar fibrosis. Due to the relatively small number of patients in this study, this group could not be classified and should be studied further.

\section{CONCLUSION}

According to HRCT manifestations and clinical pathological materials of 60 cases of children's ILD and review of previous studies, we concluded that HRCT of children's ILD could first be etiologically divided into infectious and noninfectious types. After further analysis of HRCT manifestations, children's ILD could be divided into 3 main types: 1) the early stage, which is characterized by bronchovascular bundle abnormalities that could be combined with lobular ground-glass opacities, 2) the progressive stage, which is characterized by large, patchy ground-glass opacities; although pathological changes could recover, the course of the disease was long, and 3) the bronchiectasis type, which suggested that pathological changes were irreversible.

\section{REFERENCES}

Bramson RT, Griscom NT and Cleveland RH (2005). Interpretation of chest radiographs in infants with cough and fever. Radiology 236: 22-29.

Brody AS (2008). Computed Tomography of Pediatric Small Airway Disease. Contemporary Medical Imaging: CT of the Airways. Humana Press, Totowa, 381-404.

Clement A, Nathan N, Epaud R, Fauroux B, et al. (2010). Interstitial lung diseases in children. Orphanet. J. Rare Dis. 5: 22.

Dinwiddie R, Sharief N and Crawford O (2002). Idiopathic interstitial pneumonitis in children: a national survey in the United Kingdom and Ireland. Pediatr. Pulmonol. 34: 23-29.

Fan LL and Langston C (2002). Pediatric interstitial lung disease: children are not small adults. Am. J. Respir. Crit. Care Med. 165: 1466-1467.

Farrell PM, Collins J, Broderick LS, Rock MJ, et al. (2009). Association between mucoid Pseudomonas infection and bronchiectasis in children with cystic fibrosis. Radiology 252: 534-543.

Garcia-Peña P and Lucaya J (2004). HRCT in children: technique and indications. Eur. Radiol. 14 Suppl. 4: L13-L30.

Grenier P, Valeyre D, Cluzel P, Brauner MW, et al. (1991). Chronic diffuse interstitial lung disease: diagnostic value of chest radiography and high-resolution CT. Radiology 179: 123-132.

Griese M, Haug M, Brasch F, Freihorst A, et al. (2009). Incidence and classification of pediatric diffuse parenchymal lung diseases in Germany. Orphanet. J. Rare Dis. 4: 26.

Gross TJ and Hunninghake GW (2001). Idiopathic pulmonary fibrosis. N. Engl. J. Med. 345: 517-525.

Hartl D and Griese M (2005). Interstitial lung disease in children-genetic background and associated phenotypes. Respir. Res. 6: 32.

Hilman BC (1998). Chronic interstitial lung disease. Clin. Pediatr. 37: 701-702.

Johkoh T, Muller NL, Taniguchi H, Kondoh Y, et al. (1999). Acute interstitial pneumonia: thin-section CT findings in 36 
patients. Radiology 211: 859-863.

Levine DS, Navarro OM, Chaudry G, Doyle JJ, et al. (2007). Imaging the complications of bone marrow transplantation in children. Radiographics 27: 307-324.

Long FR, Williams RS and Castile RG (2005). Inspiratory and expiratory CT lung density in infants and young children. Pediatr. Radiol. 35: 677-683.

Mayo JR, Webb WR, Gould R, Stein MG, et al. (1987). High-resolution CT of the lungs: an optimal approach. Radiology 163: 507-510.

Nambu A, Saito A, Araki T, Ozawa K, et al. (2006). Chlamydia pneumoniae: comparison with findings of Mycoplasma pneumoniae and Streptococcus pneumoniae at thin-section CT. Radiology 238: 330-338.

Park CM, Goo JM, Lee HJ, Lee CH, et al. (2007). Nodular ground-glass opacity at thin-section CT: histologic correlation and evaluation of change at follow-up. Radiographics 27: 391-408.

Seely JM, Effmann EL and Muller NL (1997). High-resolution CT of pediatric lung disease: imaging findings. AJR Am. J. Roentgenol. 168: 1269-1275.

Siegel MJ, Bhalla S, Gutierrez FR, Hildebolt C, et al. (2001). Post-lung transplantation bronchiolitis obliterans syndrome: usefulness of expiratory thin-section CT for diagnosis. Radiology 220: 455-462.

Wittram C, Mark EJ and McLoud TC (2003). CT-histologic correlation of the ATS/ERS 2002 classification of idiopathic interstitial pneumonias. Radiographics 23: 1057-1071. 